Скопје, Македонија

\title{
A CONSTRUCTION OF HEYTING ALGEBRA ON CATEGORICAL SYLLOGISMS
}

\author{
IBRAHIM SENTURK AND TAHSIN ONER
}

\begin{abstract}
The main purpose of this paper is to define a Heyting algebra on categorical syllogisms. For this aim, we explain categorical syllogisms by the diagrammatic method, which gives us a suitable treatment to logical reasoning with Caroll's diagrams. In this regard, we represent the quantitative relations between syllogisms' terms by means of bilateral diagrams. Finally, we construct a system, which is a Heyting algebra, for examining categorical syllogisms by using sets.
\end{abstract}

\section{Introduction}

The first works on categorical syllogisms go a long way back to the Aristotle [7]. From the point of Aristotle's Prior Analytics: "Syllogism is discourse in which, certain things being stated, something other than what is stated follows of necessity from their being so. I mean by the last phrase that they produce the consequence, and by this, that no further term is required from without in order to make the consequence necessary" [7].

The bases of modern mathematical references on this subject were given by Lewis Caroll[8] and Jan Eukasiewicz [6]. L. Caroll introduced an original diagrammatic scheme to examine the categorical syllogisms. Besides, he viewed his diagrammatic system which could decide some valid syllogisms. Furthermore, J. Eukasiewicz researched throughly the categorical syllogisms from the point of mathematical foundations in his book [6].

At the 19th, 20th and 21th centuries, the categorical syllogisms have been came up broadly and investigated with different treatments. For example, George Boole introduced algebraic approach to syllogistic logic in his classic "The Laws of Thought" in 1854 [4]. Stanley Burris analysed traditional syllogistic logic via Boolean algebras [11], [12] and Esko Turunen constructed an algebraic system for intermediate syllogisms by means of MV-algebras [3].

The aim of the present paper is to construct a Heyting algebra for categorical syllogisms by using sets which are obtained from bilateral diagrams.

2000 Mathematics Subject Classification. 03B80, 06D20, 18 B35.

Key words and phrases. syllogism, heyting algebra, orders and lattices. 


\section{PReliminaries}

In this part, we briefly recall some notations and terminology which will be used during this paper. The syllogism can be defined as a logical consequence which made up three categorical propositions. They consist of two premises that are called first and second premises and a conclusion which is obtained logically from these premises. Each of them has a quantified relationship between two objects. The objects in a categorical proposition are related with four different ways as follows:

TABle 1. Categorical Syllogistic Propositions

\begin{tabular}{ccc}
\hline Symbol & Statements & Generic Term \\
\hline$A$ & All $X$ are $Y$ & Universal Affirmative \\
$E$ & No $X$ are $Y$ & Universal Negative \\
$I$ & Some $X$ are $Y$ & Particular Affirmative \\
$O$ & Some $X$ are not $Y$ & Particular Negative \\
\hline
\end{tabular}

For any syllogism, the categorical propositions are composed of three terms, a subject term, a predicate term, and a middle term: the subject term is the subject of the conclusion and denoted by $S$; the predicate term modifies the subject in the conclusion and denoted by $P$, and the middle term which occurs in the two premises and links the subject and predicate terms and denoted by $M$. The subject and predicate terms occur in different premises via the middle term occurs once in each premise. The premise which consists of the predicate term and the middle term is called the major premise besides the premise which consists of subject term and the middle term is called the minor premise.

Syllogisms are grouped into four different ways depending on the position of the term-variables $S, P$ and $M$ in it as in the Table 2 .

In addition to this, each proposition in a syllogism has one of four relations. Then there are 64 different syllogistic forms for each figure. These are called moods. Hence, the categorical syllogistic system consists of 256 possible syllogisms. 24 of them are valid. However, they divide into two groups of 15 and of 9 . The first group are valid unconditionally, whereas

TABLE 2. Categorical Syllogistic Figures

\begin{tabular}{|c|c|c|c|}
\hline Major & Minor & Conclusion & Figure \\
\hline \hline$M-P$ & $S-M$ & $S-P$ & 1 \\
\hline$P-M$ & $S-M$ & $S-P$ & 2 \\
\hline$M-P$ & $M-S$ & $S-P$ & 3 \\
\hline$P-M$ & $M-S$ & $S-P$ & 4 \\
\hline
\end{tabular}


the second group are valid conditionally or valid existential import which is an explicit supposition of being of some terms.

\section{Set Theoretical Representation of Syllogistic Propositions By Using Bilateral Diagrams}

In this section, we give the set theoretical representation of syllogistic arguments by using bilateral diagrams. For this aim, we use Caroll diagrammatic method. And, we give a definition of a map which corresponds each bilateral diagram to a set.

Let $X$ and $Y$ be two terms and their complements are denoted by $X^{\prime}$ and $Y^{\prime}$, respectively. Assume that $p_{i}$ shows a possible form of any bilateral diagram, such that $1 \leq i \leq k$, where $k$ is the number of possible forms of bilateral diagram, as follows:

TABLE 3. Bilateral diagram for a quantity relation between $X$ and $Y$

\begin{tabular}{|c|c|c|}
\hline$p_{i}$ & $X^{\prime}$ & $X$ \\
\hline$Y^{\prime}$ & $n_{1}$ & $n_{2}$ \\
\hline$Y$ & $n_{3}$ & $n_{4}$ \\
\hline
\end{tabular}

where $n_{1}, n_{2}, n_{3}, n_{4} \in\{0,1\}$. Given throughout this paper the symbols $R^{(A)}, R^{(E)}, R^{(I)}$ and $R^{(O)}$ represent "All", "No", "Some" and "Somenot" statements, respectively.

Example 1. We examine "No $X$ are $Y$ ", it means that there is no element in the intersection cell of $X$ and $Y$. This situation is shown in the following bilateral diagram:

TABLE 4. Bilateral diagram for "No $X$ are $Y$ "

$$
R^{(E)}=\begin{array}{|l|l|l|}
\hline & X^{\prime} & X \\
\hline Y^{\prime} & & \\
\hline Y & & 0 \\
\hline
\end{array}
$$

From the Table 4, we obtain all possible bilateral diagrams which have 0 in the intersection cell of $X$ and $Y$ :

Table 5 shows all possible forms of "No $X$ are $Y$ ".

Now in order to correspond bilateral diagrams and sets, let us from a set consisting of numbers which correspond to possible forms that each bilateral diagram possesses. To do this, first we define the value which corresponds to the bilateral diagram. 
TABle 5. Possible forms of "No $X$ are $Y$ "

\begin{tabular}{|c|c|c|}
\hline $\boldsymbol{p}_{\mathbf{1}}$ & $X^{\prime}$ & $X$ \\
\hline$Y^{\prime}$ & 0 & 0 \\
\hline$Y$ & 0 & 0 \\
\hline
\end{tabular}

\begin{tabular}{|c|c|c|}
\hline $\boldsymbol{p}_{\mathbf{2}}$ & $X^{\prime}$ & $X$ \\
\hline$Y^{\prime}$ & 0 & 0 \\
\hline$Y$ & 1 & 0 \\
\hline
\end{tabular}

\begin{tabular}{|c|c|c|}
\hline$p_{\mathbf{3}}$ & $X^{\prime}$ & $X$ \\
\hline$Y^{\prime}$ & 0 & 1 \\
\hline$Y$ & 0 & 0 \\
\hline
\end{tabular}

\begin{tabular}{|c|c|c|}
\hline $\boldsymbol{p}_{\mathbf{4}}$ & $X^{\prime}$ & $X$ \\
\hline$Y^{\prime}$ & 1 & 0 \\
\hline$Y$ & 0 & 0 \\
\hline
\end{tabular}

\begin{tabular}{|c|c|c|}
\hline$p_{\mathbf{5}}$ & $X^{\prime}$ & $X$ \\
\hline$Y^{\prime}$ & 0 & 1 \\
\hline$Y$ & 1 & 0 \\
\hline
\end{tabular}

\begin{tabular}{|c|c|c|}
\hline$p_{\mathbf{6}}$ & $X^{\prime}$ & $X$ \\
\hline$Y^{\prime}$ & 1 & 0 \\
\hline$Y$ & 1 & 0 \\
\hline
\end{tabular}

\begin{tabular}{|c|c|c|}
\hline$p_{7}$ & $X^{\prime}$ & $X$ \\
\hline$Y^{\prime}$ & 1 & 1 \\
\hline$Y$ & 0 & 0 \\
\hline
\end{tabular}

\begin{tabular}{|c|c|c|}
\hline$p_{\mathbf{8}}$ & $X^{\prime}$ & $X$ \\
\hline$Y^{\prime}$ & 1 & 1 \\
\hline$Y$ & 1 & 0 \\
\hline
\end{tabular}

Definition 1. [1] Let $r_{j}$ be the value corresponding to a possible bilateral diagram $p_{i}$ and $n_{i}$, as defined above, that possible forms of cells may possess. Then their values are calculated by using the following formula:

$$
r_{j}=\sum_{i=1}^{4} 2^{(4-i)} n_{i}, \quad 1 \leq j \leq k,
$$

where $k$ is the number of all possible forms.

Definition 2. Let $R$ be the set of the values which correspond to all possible forms of any bilateral diagram; that is $R=\left\{r_{j}: 1 \leq j \leq k, k\right.$ is the number of all possible forms $\}$. And the set of all these $R$ 's denoted by $\mathcal{R}$.

Corollary 1. We obtain the set representations of all categorical propositions as follows:

- All $X$ are $Y$ : It means that $X$ intersection with $Y^{\prime}$ is empty set:

$$
R^{(A)}=\begin{array}{|l|l|l|}
\hline & X^{\prime} & X \\
\hline Y^{\prime} & & 0 \\
\hline Y & & \\
\hline
\end{array}
$$

From the above table, the set representation of "All $X$ are $Y$ " is

$$
R^{(A)}=\{0,1,2,3,8,9,10,11\} .
$$

- No $X$ are $Y$ :There is no element in the intersection cell of $X$ and $Y$.

$$
R^{(E)}=\begin{array}{|l|l|l|}
\hline & X^{\prime} & X \\
\hline Y^{\prime} & & \\
\hline Y & & 0 \\
\hline
\end{array}
$$

By the Example 1, we obtain

$$
R^{(E)}=\{0,2,4,6,8,10,12,14\} .
$$




$$
R^{(I)}=\begin{array}{|l|l|l|}
\hline & X^{\prime} & X \\
\hline Y^{\prime} & & \\
\hline Y & & 1 \\
\hline
\end{array}
$$

- Some $X$ are $Y$ : There is at least one element which belongs $X$ and $Y$.

By using the possible bilateral forms of $R^{(I)}$ diagram, we get

$$
R^{(I)}=\{1,3,5,7,9,11,13,15\} .
$$

- Some $X$ are not $Y$ : If some element of $X$ are not $Y$, then they have to be in $Y^{\prime}$. So, the intersection cell of $X$ and $Y^{\prime}$ is not empty. We

$$
R^{(O)}=\begin{array}{|c|c|c|}
\hline & X^{\prime} & X \\
\hline Y^{\prime} & & 1 \\
\hline Y & & \\
\hline
\end{array}
$$

obtain the below set from the $R^{(O)}$ bilateral diagram

$$
R^{(O)}=\{4,5,6,7,12,13,14,15\} .
$$

\section{Construction of Heyting Algebra on Categorical \\ SYLLOGISMS}

In this chapter, we examine some algebraic properties of categorical syllogisms by means of set theoretical representation of bilateral diagrams and we construct a Heyting algebraic system for categorical syllogisms. Firstly, we define $\wedge$ (meet) and $\vee$ (join) operators on the set of numbers corresponding to possible forms of bilateral diagrams.

Definition 3. [5] Let $R^{(1)}$ and $R^{(2)}$ be elements of $\mathcal{R}$. Then the binary join and meet operations are defined by

$$
\begin{aligned}
& R^{(1)} \vee R^{(2)}:=R^{(1)} \cup R^{(2)}, \\
& R^{(1)} \wedge R^{(2)}:=R^{(1)} \cap R^{(2)} .
\end{aligned}
$$

Lemma 1. $\langle\mathcal{R}, \vee, \wedge>$ is a distributive lattice.

Now, we define an order relation on $\mathcal{R}$ as follows:

$$
R^{(1)} \preceq R^{(2)}: \Leftrightarrow R^{(1)} \subseteq R^{(2)} .
$$

Lemma 2. $\mathcal{R}$ is a partial order set (poset) with the relation $\preceq$.

Let $(\mathcal{R}, \preceq)$ be a poset. The greatest element of $\mathcal{R}$ is $\{0,1, \ldots, 15\}$, denoted by $\mathbf{1}$ and the least element is $\emptyset$, and denoted by $\mathbf{0}$. We notice again that $\mathbf{0}$ and 0 are different from each other. Let $R$ be any element of $\mathcal{R}$. Then we obtain

$$
R \wedge \mathbf{0}=R \cap \emptyset=\emptyset=\mathbf{0}
$$


and

$$
R \vee \mathbf{1}=R \cup\{0,1, \ldots, 15\}=\{0,1, \ldots, 15\}=\mathbf{1} .
$$

Lemma 3. $<\mathcal{R}, \vee, \wedge, \boldsymbol{O}, \boldsymbol{1}>$ is a bounded lattice.

An algebraic approachto syllogistic logic was given by George Boole in The Laws of Thought [4]. He gave a suitable uniform, however, it was quite complicated to use algebraic treatment to syllogisms. But, with the help of this system, we can obtain a Heyting algebraic structure and the conclusions of syllogisms. Therefore, we can determine valid syllogisms more easily by using set of bilateral diagrams.

Definition 4. Let $R^{c}$ be the complement of $R$. Then $R^{c}=\{0,1,2, \ldots, 15\} \backslash$ $R$.

Theorem 1. $<\mathcal{R}, \vee, \wedge,{ }^{c}, \boldsymbol{O}, \mathbf{1}>$ is a Boolean algebra.

Boolean algebras were discovered as a result of Boole's investigations into the underlying laws of correct reasoning. And they have become important to electrical engineering, computer science, axiomatic set theory, model theory, and other areas of science and mathematics day by day. Our Heyting algebraic approach to categorical syllogisms will be useful for this area when they need algebraic bases in syllogistic deductions.

Definition 5. [13] An algebra $\langle\mathcal{H}, \vee, \wedge, \rightarrow, \boldsymbol{O}, \mathbf{1}>$ with three binary operations and two constants is Heyting algebra if it satisfies follows:

$\mathrm{H} 1:<\mathcal{H}, \vee, \wedge\rangle$ is distributive lattice.

$\mathrm{H} 2: x \wedge 0=0$ and $x \vee 1=1$.

H3: $x \rightarrow x=1$.

$\mathrm{H} 4:(x \rightarrow y) \wedge y=y$ and $x \wedge(x \rightarrow y)=x \wedge y$.

$\mathrm{H} 5: x \rightarrow(y \wedge z)=(x \rightarrow y) \wedge(x \rightarrow z)$ and $(x \vee y) \rightarrow z=(x \rightarrow z) \wedge(y \rightarrow$ $z)$.

For the construction of Heyting algebraic system on categorical syllogisms, we need to define $\rightarrow$ operation as below:

Definition 6. Let $\mathcal{R}$ be the set of all possible forms of syllogisms and $R^{(1)}$ and $R^{(2)}$ be elements of $\mathcal{R}$. Then the binary operation $\rightarrow$ is defined by

$$
R^{(1)} \rightarrow R^{(2)}:=\left(R^{(1)}\right)^{c} \vee R^{(2)} .
$$

Theorem 2. $<\mathcal{R}, \vee, \wedge, \rightarrow, \boldsymbol{O}, \mathbf{1}>$ is a Heyting algebra.

Proof. Axioms $H 1$ and $H 2$ follows from Lemma 1 and Lemma 2, respectively.

Let $R^{(1)}, R^{(2)}$ and $R^{(3)}$ be elements of $\mathcal{R}$. Then we have

- Axiom H3:

$$
\begin{aligned}
R^{(1)} \rightarrow R^{(1)} & :=\left(R^{(1)}\right)^{c} \vee R^{(1)}, \\
& :=\left(R^{(1)}\right)^{c} \cup R^{(1)}=\mathcal{R} .
\end{aligned}
$$


- Axiom H4:

$$
\begin{aligned}
\left(R^{(1)} \rightarrow R^{(2)}\right) \wedge R^{(2)} & :=\left(\left(R^{(1)}\right)^{c} \vee R^{(2)}\right) \wedge R^{(2)}, \\
& :=\left(\left(R^{(1)}\right)^{c} \cup R^{(2)}\right) \cap R^{(2)}, \\
& :=R^{(2)}
\end{aligned}
$$

and

$$
\begin{aligned}
R^{(1)} \wedge\left(R^{(1)} \rightarrow R^{(2)}\right) & :=R^{(1)} \wedge\left(\left(R^{(1)}\right)^{c} \vee R^{(2)}\right), \\
& :=\left(R^{(1)} \wedge\left(R^{(1)}\right)^{c}\right) \vee\left(R^{(1)} \wedge R^{(2)}\right), \\
& :=R^{(1)} \wedge R^{(2)} .
\end{aligned}
$$

- Axiom H5:

$$
\begin{aligned}
R^{(1)} \rightarrow\left(R^{(2)} \wedge R^{(3)}\right) & :=\left(R^{(1)}\right)^{c} \vee\left(R^{(2)} \wedge R^{(3)}\right) \\
& :=\left(\left(R^{(1)}\right)^{c} \vee R^{(2)}\right) \wedge\left(\left(R^{(1)}\right)^{c} \vee R^{(3)}\right) \\
& :=\left(R^{(1)} \rightarrow R^{(2)}\right) \wedge\left(R^{(1)} \rightarrow R^{(3)}\right)
\end{aligned}
$$

and

$$
\begin{aligned}
\left(R^{(1)} \vee R^{(2)}\right) \rightarrow R^{(3)} & :=\left(R^{(1)} \vee R^{(2)}\right)^{c} \vee R^{(3)} \\
:= & \left(\left(R^{(1)}\right)^{c} \wedge\left(R^{(2)}\right)^{c}\right) \vee R^{(3)}, \\
:= & \left(\left(R^{(1)}\right)^{c} \vee R^{(3)}\right) \wedge\left(\left(R^{(2)}\right)^{c} \vee R^{(3)}\right), \\
:= & \left(R^{(1)} \rightarrow R^{(3)}\right) \wedge\left(R^{(2)} \rightarrow R^{(3)}\right) .
\end{aligned}
$$

\section{REFERENCES}

[1] A. E. Kulinkovich, Algorithmization of Reasoning in Solving Geological Problems (Russian), Proceedings of the Methodology of Geographical Sciences (1979), 145161.

[2] B. A. Kulik, The Logic of Natural Discourse (Russian), Nevskiy Dialekt, (2001).

[3] E. Turunen, An Algebraic Study of Peterson's Intermediate Syllogisms, Soft Computing, vol. 18, no. 12, (2014), 2431-2444.

[4] G. Boole, The Laws of Thought, Dover Reprint., (1854).

[5] İ. Şentürk, T. Öner, U. Nuriyev. An Algebraic Approach to Categorical Syllogisms By Using Bilateral Diagrams, Theoretical and Applied Aspects of Cybernetics. Proceedings of the 5th International Scientific Conference of Students and Young Scientists, Kyiv-Ukraine), (2015), 14-21.

[6] J., Łukasiewicz, Aristotle's Syllogistic From the Standpoint of Modern Formal Logic, Oxford University Press, (1957).

[7] J. Barnes, The Complete Works of Aristotle: The Revised Oxford Translation, ed., (1984).

[8] L. Carroll, Symbolic Logic, Clarkson N. Potter, (1896).

[9] L. A. Zadeh, Syllogistic Reasoning as a Basis for Combination of Evidence in Expert Systems, 9th International Joint Conference on Artificial Intelligence, vol. 1, (1985), 417-419. 
[10] S. L. Kryvyi, A. V. Palagin, To the Analysis of Natural Languages Objects, Intelligent Processing, (2009), 36-43.

[11] S. Burris, Justifying Boole's Algebra Logic, Department of Pure Mathematics, University of Waterloo.

[12] S. Burris, A Fragment of Boole's Algebraic Logic Suitable For Traditional Syllogistic Logic, Department of Pure Mathematics, University of Waterloo.

[13] S. Burris, H. P. Sankappanavar A Course in Universal Algebra-With 36 Illustrations, (2006).

[14] T. Hailparin, Boole's Logic and Probability, Kluwer Academic Publ., (1986).

Department of Mathematics, Faculty of Science - Ege University, Turkey.

E-mail address: ibrahim.senturk@ege.edu.tr

Department of Mathematics, Faculty of Science - Ege University, Turkey.

E-mail address: tahsin.oner@ege.edu.tr 